S Nainiwal, P Garg, G Prakash and N Nainiwal

All India Institute of Medical Sciences, Dr R P Centre for Ophthalmic Sciences, Ansari Nagar, New Delhi, India

Correspondence: Dr S Nainiwal, All India Institute of Medical Sciences, Dr R P Centre for Ophthalmic Sciences, Ansari Nagar, New Delhi-29, New Delhi, Delhi 110029, India

Tel: + 911412521540 ;

Fax: + 911126588919 .

E-mail: nainvision@yahoo.com

Eye (2006) 20, 1404-1405. doi:10.1038/sj.eye.6702274; published online 24 March 2006

Sir,

\section{Bilateral vitreous haemorrhage associated with dengue} fever

We read with interest the above article by Nainiwal et al ${ }^{1}$ as it was the first report to date of vitreous haemorrhage resulting from dengue fever. A few points in the article however, require clarification and may be of relevance to the pathogenesis.

The authors have not clearly outlined the timeline of the systemic manifestations of the disease and the associated ocular symptoms and signs. The patient presented with 'diminution of vision in right eye since 4 months', 'she had high-grade fever ... 2 days before developing eye problems' and that 'she was referred to us after her general condition had improved'. We wish to clarify whether she underwent an ophthalmic assessment only 4 months after onset of dengue. Dengue haemorrhagic fever typically presents at time of defervescence some days after the onset of dengue. As such it would be useful to know when exactly from onset of the fever the patient developed ocular symptoms. Documented serum platelet levels on the day of onset of her ocular symptoms would have added further analytical value to the case report.

Recent case series have reported the mean interval between onset of visual symptoms and systemic manifestations of dengue fever to be in the range of $6.8^{2}$ and $7.3^{3}$ days. Two other case reports ${ }^{4,5}$ have reported a mean interval of 3 days. Onset of ocular symptoms around 7 days after systemic symptoms lends well to the possible immunologic basis of dengue ocular disease. $^{2}$
As it appears that the patient was not examined during the early onset of her visual symptoms, vitreous haemorrhage could have resulted at a later stage from a pre-existing dengue-related retinal haemorrhage-a known documented finding in dengue-related eye disease. $^{2}$

Dengue fever is currently endemic in Singapore and we are conducting a prospective study of ocular manifestations in patients infected with dengue virus. To date, we have had one dengue patient with bilateral vitreous haemorrhages.

However, in our case the vitreous haemorrhages were very small and not the dominant finding. Also interestingly our patient's ocular symptoms arose at the nadir of her platelet count $(29000 / \mathrm{cc})$ on day 8 of the disease. There could thus be two underlying mechanisms explaining the ocular signs in dengue eye disease. One could be immunologic with possible immune complex deposition in retinal vessels. The other could be an increased bleeding tendency resulting from decreased platelet counts.

\section{References}

1 Nainiwal S, Garg SP, Prakash G, Nainiwal N. Bilateral vitreous haemorrhage associated with dengue fever. Eye 2005; 19: 1012-1013.

2 Lim WK, Mathur R, Koh A, Yeoh R, Chee SP. Ocular manifestations of dengue fever. Ophthalmology 2004; 111: 2057-2064.

3 Wen KH, Sheu MM, Chung CB, Wang HZ, Chen CW. The ocular fundus findings in dengue fever (in Chinese). Gaoxiong Yi Xue Za Zhi 1989; 5: 24030.

4 Haritoglou C, Dotse SD, Rudolph G, Stephan CM, Thurau $\mathrm{SR}$, Klauss V. A tourist with dengue fever and visual loss. Lancet 2002; 360: 1070.

5 Cruz-Villegas V, Berrocal AM, Davis JL. Bilateral choroidal effusions associated with dengue fever. Retina 2003; 23: 576-578.

J Venkatramani and W-K Lim

Ocular Inflammation and Immunology, Singapore National Eye Centre, Singapore, Singapore

Correspondence: W-K Lim, Ocular Inflammation and Immunology,

Singapore National Eye Centre, 11,

Third Hospital Avenue,

Singapore 168751,

Singapore

Tel: + 6562277255 ; 
Fax: + 6562277290

E-mail: weekiak@cyberway.com.sg

Eye (2006) 20, 1405-1406. doi:10.1038/sj.eye.6702259; published online 3 February 2006

Sir,

Solitary nasal neurofibroma presenting as compressive optic neuropathy

Neurogenic tumour of the nasal cavity is a rare entity and it is rarer still for it to present with visual problem. We like to report a rare case of solitary nasal neurofibroma, which came to light through optic nerve compression.

\section{Case history}

A 40-year-old Malay woman was referred by the primary care physician because of blurred vision in her left inferior visual fields for the past 2 months. Her only past medical history of note was hypertension, controlled with oral atenolol. At presentation, her vision was $6 / 6$ in the right eye and 6/12 in the left eye but there was no afferent pupillary defect. The intraocular pressures in both eyes were not raised and there were no signs of glaucomatous discs. However, the left optic disc appeared pale. A visual field test was ordered which revealed a left central scotoma.

CT scan revealed a large solid midline nasal tumour (Figure 1). Superiorly the tumour eroded and left behind a thin superior wall of the sphenoid bone. However, the pituitary fossa and optic chiasm were intact. Laterally, it eroded through the ethmoid bones and sinuses. In the left orbit, the tumour can be seen to compress on the optic nerve. Inferiorly, the tumour extends into both nasal cavities and filled up the entire middle meatus. The nasal septum was destroyed. The source and nature of the tumour cannot be inferred from the scan. At the 6-week follow-up after the scan, her left vision had decreased to counting finger with an obvious relative afferent pupillary defect.

A nasal endoscopic biopsy of the lesion was performed by the ENT surgeon. The tumour appeared white with a firm consistency. Histologically, the specimen was made up of spindle wavy cells with basophilic nuclei (Figure 2). No malignant cells were seen. The initial diagnosis was that of a fibromatosis. The tumour was excised jointly by the neurosurgeon and the ENT surgeon via bifrontal craniotomy and transnasal approach,

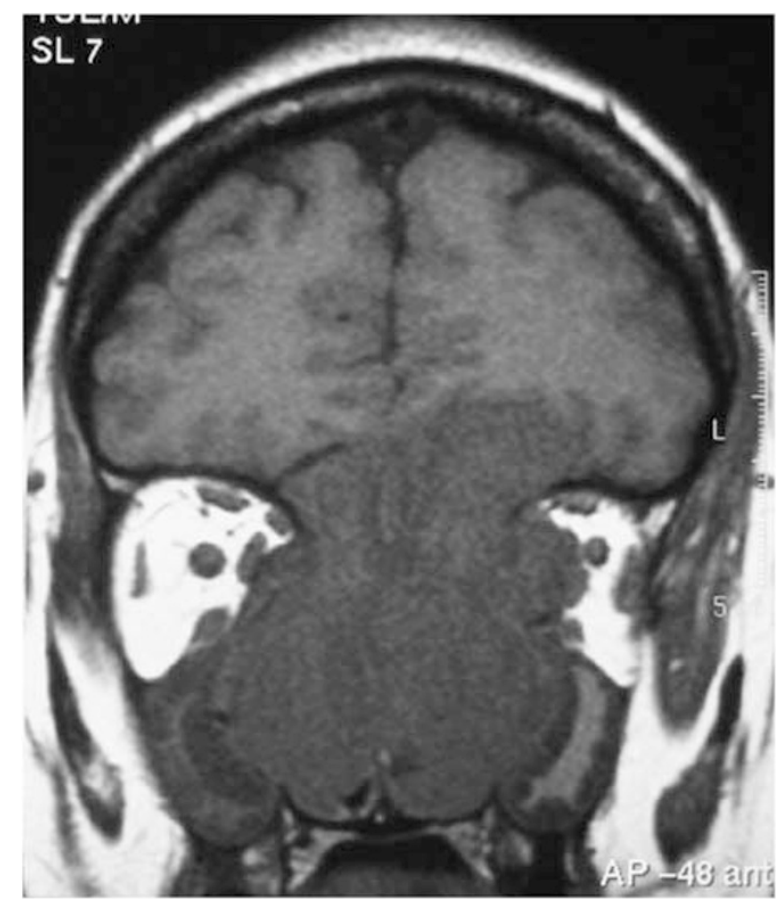

Figure 1 CT scan showing a large infiltrative midline lesion invading the left orbit and compressing the left optic nerve.

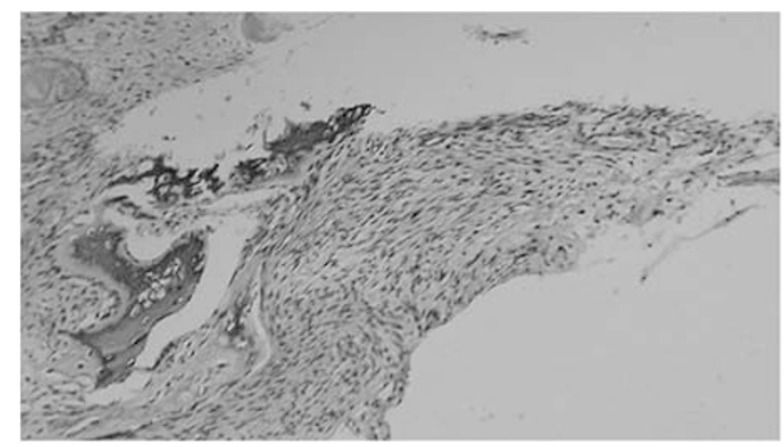

Figure 2 Biopsy specimen showing spindle wavy cells typical of neurofibromatosis (H\&E staining).

respectively. During the procedure the wall of the sphenoid and ethmoidal and the nasal septum were removed as there were infiltrated and destroyed by the lesion. Immunohistochemical studies revealed that the cells of the tumour were immunoreactive vimentin, neuron-specific enolase and S-100 protein but negative for epithelial membrane antigen. From these and the clinical findings, the tumour was diagnosed as a neurofibroma.

Postoperatively, the patient made a good recovery and her vision improved to $6 / 12$ with resolution of the relative afferent pupillary defect. Repeated serial CT scans showed the presence of residual tumour (Figure 3), which was confirmed on further nasal endoscopic biopsy. 